\title{
DeSEnVolvimento COGNITIVO E SINTOMAS DEPRESSIVOS EM ADOLESCENTES QUE FAZEM USO DE BEBIDAS ALCOÓLICAS
}

\section{Patrícia Santos Teixeira ${ }^{1}$; Maria Cristina Bergonzoni Stefanini ${ }^{2}$; Raul Aragão Martins ${ }^{3}$; Luciana Aparecida Nogueira da Cruz ${ }^{4}$}

Levantamentos sobre o uso de álcool mostram que o consumo dessa substância começa na adolescência. É comum, nessa etapa do desenvolvimento, algumas pessoas apresentarem sintomas depressivos. A combinação do uso de álcool e sintomas depressivos pode afetar o desenvolvimento cognitivo e escolar de estudantes. Esta pesquisa visa identificar e discutir a relação entre o uso de álcool pelos adolescentes, a depressão e a consequência desse uso no desenvolvimento cognitivo. Participaram da pesquisa 127 alunos de duas escolas do interior do Estado de São Paulo, que responderam ao Audit, o BDI e Provas para o Desenvolvimento Operatório. Resultados mostram que o consumo de álcool em excesso é significativo, mas não se observou relação entre o uso de álcool, sintomas depressivos e o baixo rendimento cognitivo.

Descritores: Bebidas Alcoólicas; Depressão; Consumo de Álcool; Adolescentes; Desenvolvimento Cognitivo.

\section{COGNITIVE DEVELOPMENT AND DEPRESSIVE SYMPTOMS IN ADOLESCENT ALCOHOL USERS}

Surveys on alcohol use show that people usually begin using this substance as adolescents. In this stage of development, it is common for some people to show depressive symptoms. The combination of alcohol and depressive symptoms may affect students' cognitive and school development. This study aims at identifying and discussing on the relationship between alcohol use in adolescents, depression and the consequences on cognitive development. Participants were 127 students of two schools in the state of São Paulo, who answered the AUDIT, the BDI and Tests for Operatory Development. Results show that excessive alcohol use is significant, but we did not observe a relationship between alcohol use, depressive symptoms and low cognitive performance.

Descriptors: Alcohol Beverages; Depression, Alcohol Use, Adolescents; Cognitive Development. 


\section{DESARROLLO COGNITIVO Y SÍNTOMAS DEPRESIVOS EM ADOLESCENTES QUE CONSUMEN BEBIDAS ALCOHÓLICAS}

Datos sobre el consumo de alcohol demuestran que el uso de esa sustancia comienza en la adolescencia. Es común, en esa etapa del desarrollo, que algunas personas presenten síntomas depresivos. La combinación del consumo de alcohol y síntomas depresivos puede afectar el desarrollo cognitivo y escolar de estudiantes. Esta investigación apunta a identificar y discutir la relación entre el consumo de alcohol por parte de los adolescentes, la depresión y la consecuencia del consumo en el desarrollo cognitivo. Participaron de la investigación 127 alumnos de dos escuelas del interior del estado de San Pablo, que respondieron el AUDIT, el BDI y Pruebas para el Desarrollo Operatorio. Los resultados demuestran que el consumo excesivo de alcohol es significativo, pero no observamos una relación entre ingesta de alcohol, síntomas depresivos y el bajo rendimiento cognitivo.

Descriptores: Bebidas Alcohólicas; Depresión; Uso de Alcohol; Adolescentes; Desarrollo Cognitivo.

1-Doutoranda em Educação Escolar, Faculdade de Ciências e Letras, Universidade Estadual Paulista "Júlio de Mesquita Filho", São José do Rio Preto, SP, Brasil. E-mail: pateixeira@yahoo.com.br

2-Pedagoga, Doutor em Psicologia, Professor Assistente Doutor, Faculdade de Ciências e Letras, Universidade Estadual Paulista "Júlio de Mesquita Filho", São José do Rio Preto, SP, Brasil. E-mail: alloah@gmail.com

3-Psicólogo, Doutor em Psicologia, Livre Docente, Professor Adjunto, Instituto de Biociências, Letras e Ciências Exatas, Universidade Estadual Paulista "Júlio de Mesquita Filho", São José do Rio Preto, SP, Brasil. E-mail: raul@ibilce.unesp.br

4-Psicóloga, Doutor em Educação. Instituto de Biociências, Letras e Ciências Exatas, Universidade Estadual Paulista "Júlio de Mesquita Filho", São José do Rio Preto, SP, Brasil. E-mail: lunogcruz@yahoo.com.br 


\section{Introdução}

O álcool é a droga mais consumida em todo o mundo, além de ser a de maior aceitação social. O consumo do álcool pode ser considerado, hoje, como um dos mais graves problemas de saúde pública no Brasil, e as consequências decorrentes do seu uso compreendem complicações físicas, psíquicas e sociais ${ }^{(1-2)}$. Esses problemas não envolvem apenas as pessoas que consomem o álcool em larga escala, mas toda a sociedade, pois todos têm que pagar e/ou sofrer com os danos causados pelo uso excessivo de bebida alcoólica $^{(1-2)}$.

Cabe dizer que a transição do consumo moderado e social para a dependência não é repentina, acontece com o passar dos anos e, em alguns momentos, é imperceptível para o sujeito. Outra informação importante é que o álcool pode ser uma droga de estreia e facilitadora do consumo de outras drogas. Tais informações se agravam quando estudos comprovam que o início do uso acontece, em média, aos 13 anos de idade ${ }^{(3)}$.

Os adolescentes representam um segmento da população que merece atenção, por estarem numa fase fundamental do desenvolvimento: a transição da infância para a maturidade. Nessa fase, o sujeito tem mais liberdade e maior mobilidade social. Nesse período de passagem, o jovem começa a imitar uma série de comportamentos dos adultos e, entre eles, o beber está presente ${ }^{(4-5)}$.

Os problemas emocionais enfrentados pelos jovens representam uma das causas do alto consumo de álcool. Um sujeito que esteja passando por momento de tensão, conflitos, problemas nos relacionamentos familiares ou afetivos pode consumir álcool para combater a depressão e os sentimentos indesejados. No entanto, o consumo tem efeito depressivo e só pode agravar os problemas ${ }^{(4-5)}$.

O Manual Diagnóstico e Estatístico de Transtornos Mentais-DSM-IV(6), diz que o transtorno depressivo em adolescentes traz sintomas de irritabilidade e instabilidade, podendo ocorrer crises de explosão e raiva. $\mathrm{O}$ adolescente apresenta humor irritado e perda de energia, apatia e desinteresse, retardo psicomotor, sentimentos de desesperança, culpa, perturbações do sono (principalmente hipersonia), alterações de apetite e peso, isolamento e dificuldade de concentração. Pode, também, apresentar prejuízo no desempenho escolar, baixa autoestima, queixas físicas (dor abdominal, fadiga e cefaleia), ideias e tentativas de suicídio e graves problemas de comportamento, especialmente quando há o uso abusivo de álcool e drogas.

Pesquisa $^{(7)}$ constata que a depressão em adolescentes parece estar ocorrendo cada vez mais cedo e com mais frequência, havendo maior ocorrência da doença nas meninas do que nos meninos. A taxa de depressão é baixa até os 9 anos, aumentando dos 9 aos 19 e, após os 15 anos, as meninas são 2 vezes mais afetadas que os meninos.

A presente pesquisa se pautou pela teoria do desenvolvimento cognitivo de Jean Piaget. Segundo essa teoria, os seres humanos passam por uma série de mudanças ordenadas e previsíveis de maneira interativa com o meio. Por meio da interação do sujeito com o ambiente, ocorre a construção das estruturas mentais. Assim, o sujeito interage com a realidade, interação de organização interna e adaptação ao meio, promovendo o desenvolvimento da inteligência ${ }^{(8)}$.

$\mathrm{Na}$ adolescência, o desenvolvimento cognitivo caracteriza-se pela mudança que proporciona $o$ desenvolvimento progressivo de ideias. É o momento em que os jovens constroem sistemas e teorias. O sujeito revela seu desejo de se integrar em um mundo diferente. Para o sistema educacional, o desenvolvimento cognitivo é avaliado a partir de seu rendimento escolar que, quando bem-sucedido, traz perspectivas para o futuro, e o seu fracasso bloqueia essas perspectivas ${ }^{(9)}$.

Não se pode afirmar que o álcool, associado a sintomas depressivos, é agente causador de problemas no desenvolvimento escolar de seus consumidores. Porém, acredita-se que, em associação com outros fatores, pode contribuir para o rendimento cognitivo não satisfatório, principalmente associado às modificações que o adolescente sofre na passagem da infância para a fase adulta ${ }^{(10)}$.

Embora existam pesquisas mostrando padrões de uso de álcool e outras drogas entre adolescentes, sabe-se pouco a respeito daquilo que pode levá-los a consumir mais álcool e as consequências sobre seu desenvolvimento emocional e cognitivo ${ }^{(11-12)}$. O desafio, neste estudo, é entender os problemas relacionados ao consumo de álcool e suas consequências e influências no comportamento do bebedor. Dessa forma, neste trabalho o objetivo é identificar e discutir a relação entre o uso abusivo de álcool dos adolescentes escolarizados, a depressão e a consequência desse uso no desenvolvimento cognitivo. Entender o que mantém o consumo e as consequências para a vida do jovem é importante para a implantação de políticas preventivas quanto ao consumo de álcool por adolescentes.

Outra razão, pela qual este estudo também se justifica, é a escassez de pesquisas científicas relacionadas ao objeto proposto na presente investigação. E, ainda, por se acreditar que o reconhecimento das características dos usuários é necessário para que este estudo possa contribuir com a literatura sobre o assunto, ajudando os profissionais tanto da educação quanto da saúde a lidar com essa problemática.

\section{Método}

A pesquisa constou de duas etapas interdependentes.

Etapa 1 - O levantamento inicial, com a realização da identificação dos alunos do primeiro ano do ensino médio. Optou-se por alunos do primeiro ano, porque pesquisas sobre o padrão de consumo de álcool entre adolescentes vêm mostrando que a idade média, para o início do consumo, varia em torno de 12 a 13,5 $\operatorname{anos}^{(3-11)}$, além de se mostrarem mais disponíveis e menos preocupados com o vestibular.

Etapa 2 - A entrevista, resultou na classificação do desenvolvimento cognitivo dos sujeitos.

A pesquisa foi realizada em duas escolas de uma cidade com cerca de 400 mil habitantes, localizada na 
região noroeste do Estado de São Paulo. A primeira escola é uma instituição pública, localizada em um bairro de classe média, com 787 alunos matriculados no ensino médio, sendo 236 deles no primeiro ano. $\mathrm{O}$ levantamento inicial foi aplicado em 85 alunos sorteados, aleatoriamente, representando $36 \%$ dos alunos do primeiro ano do ensino médio dessa escola. A segunda escola é uma instituição privada, organizada como cooperativa sem fins lucrativos, de educação infantil, ensino fundamental e médio, localizada em bairro de classe média alta da cidade, com o total de 117 alunos matriculados no ensino médio. Participaram do levantamento inicial todos os alunos do primeiro ano do ensino médio da escola particular, totalizando 47 alunos. Tais escolas foram escolhidas pela facilidade de acesso, disponibilidade e a semelhança no grupo social (classe média).

Instrumentos da Etapa 1

O teste de identificação de desordens devido ao uso de álcool - Audit ${ }^{(13)}$ - avalia o padrão do beber entre jovens e adultos, e sua pontuação varia de zero a 40 pontos. Os alunos que pontuaram até sete foram chamados abstêmios, ou que bebem moderadamente, e aqueles que atingiram oito ou mais pontos representam aqueles que consomem em excesso.

O Audit foi escolhido para compor o levantamento inicial pela necessidade de se ter um instrumento com sensibilidade alta para alcançar o máximo de sujeitos passíveis de participar da entrevista, referente à segunda etapa da pesquisa. Além disso, é de preenchimento rápido e já foi utilizado com população de adolescentes e jovens. Esse instrumento foi desenvolvido por um grupo de pesquisadores e já está adaptado para o Brasil ${ }^{(13)}$, sob os auspícios da Organização Mundial da Saúde - $\mathrm{OMS}^{(14)}$.

O segundo instrumento é referente ao nível de depressão dos adolescentes e foi utilizado o inventário para depressão BDI (BDI - Beck Depression Inventory) (15), instrumento de autopreenchimento, composto por 21 itens que avalia a sintomatologia presente nas últimas semanas. Foi extensamente validado em amostras clínicas e populacionais brasileiras que encontraram pontos de corte para diferentes intensidades dos sintomas depressivos: mínimo (0-11), leve (12-19), moderado (20-35) e grave (36 a 63). No presente estudo, utilizou-se a nota de corte maior ou igual a 12 para identificação de casos de sujeitos com sintomas depressivos) $)^{(15)}$.

Esse levantamento possibilitou identificar os participantes que consumiam bebidas alcoólicas em excesso e aqueles que faziam uso moderado ou eram abstêmios $^{(16)}$. Também foram identificados os grupos de adolescentes com sintomas depressivos e, a partir daí, formaram-se quatro grupos.

Instrumento da Etapa 2

Para a avaliação do desenvolvimento cognitivo dos sujeitos, foram escolhidas três provas operatórias para o pensamento formal: prova de combinação de fichas duplas para pensamento formal, permutações possíveis com um conjunto determinado de fichas (para o pensamento formal) e Torre de Hanói.

As "provas clássicas" formam os instrumentais elaborados por Piaget em suas pesquisas com a equipe de Genebra, objetivando determinar o grau de aquisição de algumas noções chave do desenvolvimento cognitivo, detectando o nível de pensamento alcançado pela criança ou jovem, ou seja, o nível de estrutura cognitiva com que opera. É necessário sublinhar que as provas clássicas de Piaget, utilizadas na presente pesquisa, foram, originalmente, formuladas para uma população europeia e ainda não foram validadas para o contexto nacional. A descrição detalhada delas é encontrada em inúmeras obras sobre a teoria piagetiana e, originalmente, nos textos de Piaget e colaboradores, dentro de seus relatórios de pesquisas sobre a Epistemologia Genética. Para efeito desta pesquisa, utilizar-se-á a sistematização feita pelo próprio autor e colaboradores ${ }^{(17-18)}$.

\section{Participantes da entrevista - Etapa 2}

Do grupo de 127 estudantes que responderam ao Levantamento Inicial, 21 (16,5\%) deles fizeram oito ou mais pontos no Audit e foram classificados como "Positivos". Isso significa que esses estudantes apresentam beber de risco ou excessivo. Os demais, que fizeram entre zero e sete pontos, foram classificados como "Negativos", ou seja, são abstêmios ou bebem moderadamente. Com a utilização do instrumento BDI, participaram da segunda fase 20 estudantes que foram submetidos à avaliação de desenvolvimento cognitivo. O grupo foi composto por $65 \%$ de estudantes do sexo masculino e $35 \%$ de estudantes do sexo feminino. Com relação à faixa etária, todos os sujeitos eram adolescentes com idade entre 14 e 16 anos e a maioria (60\%) deles tinha 15 anos.

Esta amostra correspondeu a cerca de $50 \%$ do grupo positivo. A maior parte dos participantes positivos era do sexo masculino, matriculados no período noturno.

A partir dos resultados da primeira etapa, os sujeitos foram organizados em 4 grupos: alunos que consomem álcool em excesso e não apresentam depressão (grupo 1), alunos que não consomem álcool e não apresentam depressão (grupo 2), alunos que consomem álcool em excesso e apresentam depressão (grupo 3) e o grupo de alunos que não consome álcool e apresenta depressão (grupo 4). Julgou-se importante a caracterização dos grupos para melhor traçar o perfil do jovem nesse momento.

\section{Análise dos Dados}

Todos os dados foram digitados em planilha eletrônica e, posteriormente, exportados para um programa de análises estatísticas ${ }^{(19)}$. Nesse programa, foram computados, em um primeiro momento, as frequências de cada questão e observada a presença de valores não esperados, para, posteriormente, serem realizadas análises mais detalhadas.

As três provas foram avaliadas quanto à fidedignidade em seus procedimentos avaliativos (ausência de capacidade 
operatória - nível 1; condutas intermediárias - nível 2 e condutas operatórias revelando capacidade combinatória - nível 3).

\section{Resultados}

Os resultados gerais são apresentados na mesma sequência da aplicação dos testes. Primeiro, aqueles relativos ao consumo de álcool, em seguida a ocorrência da depressão e, por último, o nível de desenvolvimento cognitivo dos participantes.

\section{O consumo de álcool}

Os resultados relativos ao uso de álcool e a depressão são apresentados na Tabela 1 , na qual se pode observar que $16,5 \%$ dos alunos pontuaram oito ou mais no teste Audit, com pequena diferença entre os alunos das escolas pública e particular.

No que se refere ao sexo, encontrou-se $4,7 \%$ de

Tabela 1 - Consumo de álcool por escola

\begin{tabular}{lcccccc}
\hline \multirow{2}{*}{ Escola } & \multicolumn{2}{c}{ Positivo } & & \multicolumn{2}{c}{ Negativo } \\
\cline { 2 - 3 } \cline { 6 - 7 } & $\mathbf{f}$ & $\%$ & & $\mathbf{f}$ & $\%$ \\
\hline Particular & 7 & 16,3 & & 36 & 83,7 \\
Pública & 14 & 16,7 & & 70 & 83,3 \\
Total de alunos & 21 & 16,5 & & 106 & 83,5 \\
\hline
\end{tabular}

meninas que consomem álcool em excesso e 11,8\% de meninos. Todos passaram a ser considerados pertencentes ao grupo dos que usam álcool em excesso. Esses resultados reforçam outras pesquisas que mostram que os meninos são os maiores consumidores ${ }^{(20-21)} \mathrm{em}$ excesso, chegando, em pesquisa recente, a $66,7 \%$ do grupo pesquisado.

Alguns estudos mostram que existem mais adultos bebendo em excesso do que adolescentes e que, portanto, o consumo excessivo aumenta com o passar do tempo, ou seja, a prevalência do uso cresce linearmente com a idade $^{(20-21)}$.

\section{Incidência de comportamento depressivo}

Nos resultados relativos ao comportamento depressivo, pode-se identificar que $84,3 \%$ dos sujeitos apresentam comportamento depressivo mínimo, ou seja, apenas algum comportamento depressivo em algum momento, enquanto $15,8 \%$ estão apresentando comportamento depressivo, sendo $17 \%$ da escola pública e apenas 3\% da escola particular.

Em relação ao sexo, foi observada a tendência feminina para a depressão. Enquanto os meninos apresentam 4,3\% de comportamento depressivo, as meninas dobram esse número, com $8,7 \%$.

No cruzamento das informações sobre álcool e

Tabela 2 - Resultados do comportamento depressivo por escola

\begin{tabular}{ccccccc}
\hline \multirow{2}{*}{ Escola } & \multicolumn{2}{c}{ Mínima } & & \multicolumn{2}{c}{ Com depressão } \\
\cline { 2 - 3 } \cline { 6 - 6 } \cline { 5 - 6 } Particular & $\mathbf{f}$ & $\%$ & & $\mathbf{f}$ & $\%$ \\
Pública & 40 & 93,0 & & 3 & 7,0 \\
Total & 67 & 70,8 & & 17 & 20,2 \\
\hline
\end{tabular}

comportamento depressivo, apresentadas na Tabela 3, encontraram-se 15 alunos que apresentam resultado positivo para depressão e resultado negativo para uso de álcool, enquanto apenas cinco alunos são positivos para álcool e depressão. Esse resultado auxiliou, aqui, para a escolha do número de sujeitos por grupos, na segunda fase da pesquisa.

Tabela 3 - Cruzamento entre alunos que fazem uso abusivo de álcool e que apresentam comportamento depressivo

\begin{tabular}{lcccccc}
\hline \multirow{2}{*}{ Beck } & \multicolumn{4}{c}{ Audit } \\
\cline { 2 - 3 } \cline { 6 - 7 } & \multicolumn{2}{c}{ Negativo } & & \multicolumn{2}{c}{ Positivo } \\
\cline { 2 - 3 } \cline { 5 - 6 } & $\mathbf{f}$ & $\%$ & & $\mathbf{f}$ & $\%$ \\
\hline Depressão mínima & 91 & 85,0 & & 16 & 15,0 \\
Depressão & 15 & 75,0 & & 5 & 25,0 \\
\hline
\end{tabular}

\section{Desenvolvimento Cognitivo}

Esta categoria é composta pela aplicação das provas operatórias piagetianas, as quais permitem a classificação em níveis. A primeira prova consistiu na prova de combinação das fichas duplas para pensamento formal. Nessa prova, encontraram-se três sujeitos nas condutas intermediárias. Entretanto, a maioria, 17 sujeitos, apresentava capacidade combinatória, alcançando o nível 3, que significa a presença do pensamento abstrato (Tabela 4).

A segunda prova foi de permutações com um conjunto determinado de fichas. Nessa prova, encontrou-se, no total, 35\% dos sujeitos, sete, em condutas intermediárias e $65 \%$ em condutas de realizações de todas as permutações possíveis, alcançando o nível 3, que significa a presença do pensamento abstrato.

$\mathrm{Na}$ terceira prova, com a Torre de Hanói, encontrouse um (5\%) sujeito no nível 1, 30\% dos sujeitos no nível intermediário e $65 \%$ no nível 3 , que significa a presença do pensamento abstrato. Nessa prova, foram encontrados alguns resultados contraditórios. Isso parece se dever ao fato de que os sujeitos apresentam êxito empírico sem a demonstração da lei, ou seja, realizam a atividade, porém, não compreendem a regra abstrata capaz de explicar o resultado obtido na tarefa.

Tabela 4 - Resultados das provas operatórias piagetianas por sujeitos

\begin{tabular}{|c|c|c|c|c|c|c|}
\hline \multirow{3}{*}{ Nível } & \multicolumn{6}{|c|}{ Provas } \\
\hline & \multicolumn{2}{|c|}{ Combinação } & \multicolumn{2}{|c|}{ Permutação } & \multicolumn{2}{|c|}{ Torre de Hanó } \\
\hline & f & $\%$ & $f$ & $\%$ & f & $\%$ \\
\hline 1 & 0 & 0 & 0 & 0 & 1 & 5 \\
\hline 2 & 3 & 15 & 7 & 35 & 6 & 30 \\
\hline 3 & 17 & 85 & 13 & 65 & 13 & 65 \\
\hline Total & 20 & 100 & 20 & 100 & 20 & 100 \\
\hline
\end{tabular}

Caracterização dos grupos

Apresenta-se, abaixo, a visualização do desempenho operatório de cada grupo (Tabela 5) e seus respectivos comentários. 
Tabela 5 - Visualização do desempenho operatório dos grupos 1, 2, 3 e 4

\begin{tabular}{|c|c|c|c|c|c|c|}
\hline & \multicolumn{6}{|c|}{ Provas } \\
\hline & \multicolumn{2}{|c|}{ Combinação } & \multicolumn{2}{|c|}{ Permutação } & \multicolumn{2}{|c|}{$\begin{array}{c}\text { Torre de de } \\
\text { Hanói }\end{array}$} \\
\hline & $f$ & $\%$ & $f$ & $\%$ & $f$ & $\%$ \\
\hline \multicolumn{7}{|l|}{ Grupo 1} \\
\hline Nível 1 & 0 & 0 & 0 & 0 & 0 & 0 \\
\hline Nível 2 & 1 & 20 & 2 & 40 & 1 & 20 \\
\hline Nível 3 & 4 & 80 & 3 & 60 & 4 & 80 \\
\hline Total & 5 & 100 & 5 & 100 & 5 & 100 \\
\hline \multicolumn{7}{|l|}{ Grupo 2} \\
\hline Nível 1 & 0 & 0 & 0 & 0 & 1 & 20 \\
\hline Nível 2 & 1 & 20 & 2 & 40 & 3 & 60 \\
\hline Nível 3 & 4 & 80 & 3 & 60 & 1 & 20 \\
\hline Total & 5 & 100 & 5 & 100 & 5 & 100 \\
\hline \multicolumn{7}{|l|}{ Grupo 3} \\
\hline Nível 1 & 0 & 0 & 0 & 0 & 0 & 0 \\
\hline Nível 2 & 0 & 0 & 1 & 20 & 2 & 40 \\
\hline Nível 3 & 5 & 100 & 4 & 80 & 3 & 60 \\
\hline Total & 5 & 100 & 5 & 100 & 5 & 100 \\
\hline \multicolumn{7}{|l|}{ Grupo 4} \\
\hline Nível 1 & 0 & 0 & 0 & 0 & 0 & 0 \\
\hline Nível 2 & 1 & 20 & 2 & 40 & 0 & 0 \\
\hline Nível 3 & 4 & 80 & 3 & 60 & 5 & 100 \\
\hline Total & 5 & 100 & 5 & 100 & 5 & 100 \\
\hline
\end{tabular}

Grupo 1 (positivo álcool e negativo comportamento depressivo)

O grupo 1 é composto $100 \%$ pelo sexo masculino, $80 \%$ dos sujeitos apresenta nível 3 para conduta operatória, revelando capacidade operatória, $80 \%$ apresenta nível 3 para conduta de realizações de todas as permutações possíveis, enquanto $40 \%$ se encontra no nível 2 com permutas intermediárias. E $80 \%$ dos sujeitos apresenta nível 3 para conduta de realizações dedutivas e elaboração da lei matemática $2^{\mathrm{n}}-1$ :

De modo geral, não foi encontrada relação entre uso de álcool e baixo desempenho cognitivo, já que a maioria do grupo apresenta nível 3. Exceção representada pelo sujeito de número 70, que apresenta condutas intermediárias. Os resultados mostram que os adolescentes que fazem uso de álcool apresentam desenvolvimento cognitivo esperado.

Grupo 2 (negativo álcool e negativo comportamento depressivo)

O grupo 2 é constituído $60 \%$ pelo sexo masculino, $80 \%$ dos sujeitos apresentam nível 3 para conduta operatória, revelando capacidade operatória, 60\% apresenta nível 3 para conduta de realizações de todas as permutações possíveis, enquanto apenas $20 \%$ se encontra no nível 3 , com a elaboração da lei matemática $2^{\mathrm{n}}-1$.

O grupo 2 não apresenta resultado positivo para uso de álcool ou depressão, no entanto, a maioria do grupo apresenta rendimento cognitivo intermediário.

Grupo 3 (positivo álcool e positivo comportamento depressivo)

Esse grupo é composto $60 \%$ pelo sexo masculino, $100 \%$ dos sujeitos apresentam nível 3 para conduta operatória, revelando capacidade operatória, $80 \%$ apresenta nível 3 para conduta de realizações de todas as permutações possíveis, enquanto $40 \%$ se encontra no nível 2, com permutas intermediárias. E $60 \%$ dos sujeitos apresentam nível 3 para conduta de realizações dedutivas e elaboração da lei matemática $2^{\mathrm{n}}-1$.

O grupo 3 é positivo para depressão e uso de álcool, no entanto, a maioria do grupo, 60\%, apresenta ótimo rendimento cognitivo.

Grupo 4 (negativo álcool e positivo comportamento depressivo)

O grupo 4 é composto $80 \%$ pelo sexo feminino, $80 \%$ dos sujeitos apresentam nível 3 para conduta operatória, revelando capacidade operatória, 40\% apresenta nível 2 para conduta de realizações de permutações intermediárias, enquanto $60 \%$ se encontra no nível 3 , com permutações possíveis. E $100 \%$ dos sujeitos apresentam nível 3 para conduta de realizações dedutivas e elaboração da lei matemática $2^{\mathrm{n}}-1$.

O grupo 4 é positivo para depressão e negativo para uso de álcool, e a maioria do grupo, $60 \%$, apresenta ótimo rendimento cognitivo.

O grupo 4 consome álcool em baixa quantidade e pode ser observado que todos relatam terem passado por experiências nas quais as pessoas próximas (família e amigos) consomem álcool em excesso e não refletem uma boa imagem.

\section{Conclusões}

Diante dos resultados obtidos na presente pesquisa, levantaram-se algumas hipóteses, pois não se observa relação entre o uso de álcool, o comportamento depressivo e o desenvolvimento cognitivo. Os resultados não corresponderam ao esperado - consumo excessivo de álcool e baixo rendimento cognitivo. Colocou-se como primeira hipótese o fato de a amostra estudada ter sido pequena e, talvez, por essa razão, os números não sejam significativos. Outra hipótese é que as consequências da relação entre consumo de substâncias psicoativas e déficit cognitivo são tardias, ou seja, os efeitos do álcool podem ser futuramente prejudiciais ao estudante. Só uma pesquisa longitudinal, nesse caso, poderia confirmar essa hipótese.

Foram encontrados resultados nos quais os sujeitos apresentam rendimento cognitivo (nível esperado) nível 3 e, no entanto, suas notas são "vermelhas". Sabe-se que o processo de tomada de consciência das operações cognitivas dedutivas está em elaboração, mesmo que se trate de sujeitos de 14 a 16 anos de idade. Questionou-se como a escola percebe esse processo, já que o rendimento desses alunos não é elevado. Porém, os resultados nas provas operatórias apontam que esses sujeitos possuem capacidade cognitiva para melhor desempenho nas disciplinas escolares, o que não acontece na prática.

Em atenção ao caráter preventivo dessa pesquisa, salienta-se, ainda, duas informações importantes colhidas das declarações dos próprios estudantes: os mesmos afirmam beber em família, mas afirmam não comprar o produto, recebendo-o de outra pessoa.

A partir das informações desta pesquisa, programa de redução de danos em relação ao uso de álcool não pode deixar de considerar os papéis e as ações da escola e da 
família, ou seja, qualquer programa não pode desconhecer que o bebedor jovem consome em família, e que a escola pode não estar aproveitando e estimulando seu potencial cognitivo de maneira adequada.

\section{Referências}

1. Ministério da Saúde(BR). Secretaria executiva, Secretaria de atenção a Saúde, Coordenação Nacional DST/AIDS. A política do ministério da saúde para a atenção integral a usuários de álcool e outras drogas. Brasília: Ministério da Saúde; 2003. [acesso 10 ago 2009] Disponível em: http:// bvsms.saude.gov.br/bvs/publicações/pns_alcool_drogas. pdf

2. Ministério da Saúde (BR). Secretaria Executiva, Secretaria de atenção a Saúde. Departamento de Ações Programáticas Estratégicas. Álcool e redução de danos: uma abordagem inovadora para países em transição/Ministério da Saúde/ Secretaria de atenção á saúde. Departamento de Ações Programáticas Estratégicas. Brasília: Ministério da Saúde; 2004. [acesso 10 ago 2009] Disponível em: http:// portal.saude.gov.br/portal/arquivos/pdf/A\%20politica.pdf 3. Martins RA, Manzato AJ, Cruz LAN. O uso de bebidas alcoólicas entre adolescentes. In: Castro LR, Correa J. Juventude contemporânea: perspectivas nacionais e internacionais. Rio de Janeiro: NAU Editora: FAPERJ; 2005. p. 301-26.

4. Fishman R. Alcoolismo. Coleção tudo sobre drogas. São Paulo: Nova Cultural; 1988.

5. Vieira DL, Ribeiro M, Romano M, Laranjeira RR. Álcool e adolescentes: estudo para implementar políticas municipais. Rev Saúde Pública. jun 2007;41(3):396403. [acesso 29 set 2010] Disponível em: http://www. scielo.br/scielo.php?script=sci_arttext\&pid=S003489102007000300011\&lng=pt. Epub 29-Mar-2007. doi: 10.1590/S0034-89102006005000022

6. Batista D. Manual Diagnóstico e Estatístico de Transtornos Mentais. Porto Alegre: Artes Médicas, 1995.

7. Bahls SC, Depressão na adolescência: características clínicas. Rev Interação Psicol. 2002;6(1):49-57.

8. Piaget J. O nascimento da inteligência na criança. Rio de Janeiro: Zahar; 1970.

9. Barros APR, Coutinho MPL, Araújo LF, Castanha AR. As representações sociais da depressão em adolescentes no contexto do ensino médio. Estudos Psicol. 2006;23(1):1928.

10. Teixeira PS. Adolescente, uso de álcool, depressão e desenvolvimento cognitivo. 2007. [Dissertação Mestrado em Educação Escolar]. Araraquara: Faculdade de Ciências e Letras da Universidade Estadual Paulista; 2007.

11. Tavares BF, Béria JU, Lima MS. Fatores associados ao uso de drogas entre adolescentes escolares. Rev Saúde Pública. 2004;38(6):787-96.

12. Guimarães JL, Godinho PH, Cruz LAN, Kappann JI, Junior LAT. Consumo de drogas psicoativas por adolescentes escolares de Assis, SP. Rev Saúde Pública. 2004;38(1):130-2.

13. Méndez EB. Uma versão brasileira do AUDIT Alcohol Use Disorders Identification Test. [Dissertação de Mestrado]. Pelotas: Universidade Federal de Pelotas;. 1999.

14. Babor TF, Higgins-Biddle JC, Saunders JB, Monteiro MG. AUDIT - The alcohol use disorders identification test: guidelines for use in primary health care. Genebra: World Health Organization/PAHO-92; 1992. n. 4, p. 1-29.

15. Cunha JÁ. Manual da versão em português da Escala Beck, São Paulo: Casa do Psicólogo Livraria e Editora; 2001. p. 1-171.

16. Martins RA, Cruz LAN, Teixeira PS, Manzato AJ. Padrão de consumo de álcool entre estudantes do ensino médio de uma cidade do interior do estado de São Paulo. SMAD, Rev Eletrônica Saúde Mental Álcool Drog. (Ed. port.); fev 2008.;4(1). [acesso maio 2008]. Disponível em: http://pepsic.bvs-psi.org.br/scielo.php?script=sci_ arttext\&pid=S1806-69762008000100005\&lng=pt\&nrm= 17. Piaget J, A tomada de consciência. São Paulo: Melhoramentos; EDUSP; 1977.

18. Weiss ML. Psicopedagogia Clínica. Rio de Janeiro: DP\&A; 2003.

19. SPSS. SPSS, Versão 12. Chicago: SPSS Inc; 2003.

20. Cruz LAN. Uso de álcool e julgamento sócio-moral de estudantes do ensino médio. 2006. [Dissertação de Mestrado em Educação]. Marília: Faculdade de Filosofia e Ciências da Universidade Estadual Paulista; 2006.

21. Martins RA. Uso de álcool, intervenção breve e julgamento sócio-moral em adolescentes que bebem excessivamente. [Tese Livre-Docência em Psicologia da Educação]. São José do Rio Preto: Instituto de Biociências e Ciências Exatas, Universidade Estadual Paulista de São José do Rio Preto; 2006. 211 f.

\section{Como citar este artigo:}

Teixeira PS, Stefanini MCB, Martins RA, Cruz LAN. Desenvolvimento cognitivo e sintomas depressivos em adolescentes que fazem uso de bebidas alcoólicas. SMAD, Rev. Eletrônica Saúde Mental Álcool Drog. (Ed. port.). jan-abr 2011 [acesso: ];7(1):3-9. Disponível em:

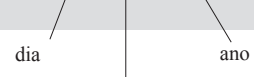

mês abreviado com ponto 